\title{
Clinicopathologic Characteristics and Outcomes of Simultaneous Multiple Primary Lung Cancer
}

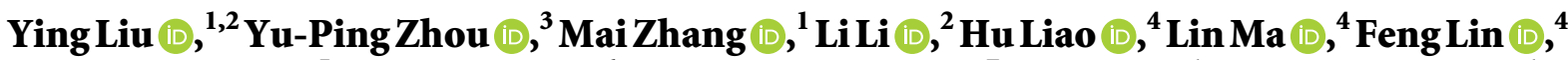 \\ Yue-Yun Chen $\mathbb{D},{ }^{5}$ Chun-Xi Fu $\mathbb{D}^{\mathbb{D}},{ }^{6}$ Ting-Ting Huang $\mathbb{D}^{7},{ }^{7}$ You Lu $\mathbb{D},{ }^{1}$ and Yan Zhang $\mathbb{D}^{1}$ \\ ${ }^{1}$ Department of Thoracic Oncology, State Key Laboratory of Biotherapy and Cancer Center, West China Hospital, \\ Sichuan University, Chengdu, Sichuan, China \\ ${ }^{2}$ The Comprehensive Cancer Center of Drum Tower Hospital, Clinical Cancer Institute of Nanjing University, Nanjing, China \\ ${ }^{3}$ Department of Cardiology, State Key Laboratory of Complex Severe and Rare Diseases, Peking Union Medical College Hospital, \\ Chinese Academy of Medical Science, Peking Union Medical College, Beijing, China \\ ${ }^{4}$ Department of Thoracic Surgery, West China Hospital, Sichuan University, Chengdu, Sichuan, China \\ ${ }^{5}$ Department of Biotherapy, Cancer Center, West China Hospital, West China Medical School, \\ State Key Laboratory of Biotherapy, Sichuan University, Chengdu, Sichuan, China \\ ${ }^{6}$ Department of Pediatric Hematology and Oncology, West China Second University Hospital, Sichuan University, Chengdu, \\ Sichuan, China \\ ${ }^{7}$ Department of Pneumology, Peking Union Medical College Hospital, \\ Chinese Academy of Medical Science and Peking Union Medical College, Beijing, China
}

Correspondence should be addressed to Yan Zhang; zhang.yan@scu.edu.cn

Received 20 June 2021; Accepted 30 November 2021; Published 23 December 2021

Academic Editor: Luca Cantini

Copyright $\odot 2021$ Ying Liu et al. This is an open access article distributed under the Creative Commons Attribution License, which permits unrestricted use, distribution, and reproduction in any medium, provided the original work is properly cited.

Background. Simultaneous multiple primary lung cancer has been detected increasingly nowadays with the development of image technology. However, the clinicopathologic characteristics and outcomes are not clear. Methods. All consecutive patients diagnosed as simultaneous multiple primary lung cancer according to Martini-Melamed and American College of Chest Physicians criteria from June 2010 to June 2019 in our center were enrolled. The clinicopathologic characteristics and outcomes were compared between patients with the same histological type and different histological types. Results. A total of 336 patients were enrolled, consisting of 297 (88.4\%) patients with the same histological type and $39(11.6 \%)$ patients with different histological types. Compared to patients with the same histological type, patients with different histological types were more commonly males ( $87.2 \%$ vs. $34.0 \%$; $p<0.001)$ with an older age $(65$ [62-69] vs. 59 [52-65] yrs; $p<0.001)$ at diagnosis. Also, patients with different histological types showed worse respiratory function and more advanced stage according to TNM staging. The 1-, 2-, and 3-year overall survival of overall patients was 97.7\%, 96.1\%, and 92.2\%, and the 1-, 2-, and 3-year recurrence-free survival of overall patients was $96.8 \%, 92.9 \%$ and $85.7 \%$, respectively. Importantly, patients with different histological types showed worse overall survival $(p<0.001)$ and recurrence-free survival $(p=0.002)$ than patients with same histological type. The multivariable Cox proportional hazard model revealed that presence of different histological types was significant predictor for worse overall survival (adjusted hazard ratio: 10.00; 95\% confidence interval: $2.92-34.48 ; p<0.001$ ) and recurrence-free survival (adjusted hazard ratio: 2.59; $95 \%$ confidence interval: 1.14-5.88; $p=0.023$ ). Conclusions. Although relatively less common in simultaneous multiple primary lung cancer, patients with different histological types showed worse clinical characteristics and outcomes.

\section{Introduction}

Multiple primary lung cancer (MPLC) refers to the occurrence of two or more primary lung cancers in one or both lungs at the same time or successively. According to the time interval of different cancer lesions, MPLC was divided into simultaneous MPLC (sMPLC) and metachronous MPLC (mMPLC). The criteria for the diagnosis of MPLC was first established by Martini and Melamed [1] and later developed by the American College of Chest Physician (ACCP) [2]. 
Both criteria are based on clinicopathologic and radiological features of lung nodules and have been in mainstream use due to their practicality, especially for preoperative evaluation. Notably, recent attempts of incorporating molecular and histological profiles into the diagnosis suggested their superiorities in distinguishing between MPLC and intropulmonary metastasis, which showed promising prospects [3-6].

With the use of high-resolution chest imaging system and lung cancer screening program, patients with MPLCs are becoming a growing population in clinical practice worldwide, especially for sMPLC [7-9]. In patients with sMPLC, the histological types of different lesions are critical and patients can be divided into two groups, including patients with the same histological type and patients with different histological types. There were some studies previously reported the proportion of patients with different histological types in sMPLC. However, due to the limited sample size and different population of reported studies, the proportion ranged from $3.8 \%$ to $61.5 \%$ in reported studies [10-13]. In addition, there was rare evidence about the differences of clinicopathologic characteristics and outcomes between patients with the same histological type and different histological types.

So, the aim of the present study was to investigate the proportion of patients with different histological types in sMPLC and confirm the differences of clinicopathologic characteristics and outcomes between patients with the same histological type and different histological types.

We present the following article in accordance with the STROBE Reporting Checklist.

\section{Methods}

2.1. Study Design and Participants. From June 2010 to June 2019, all consecutive patients diagnosed as sMPLC after surgery resection in our center according to Martini-Melamed [1] and American College of Chest Physicians criteria [2] were enrolled in this cohort study. All resected lesions were conducted with pathological examination to investigate the histological type. These patients were classified into two groups according to the histological types of lesions from the same patient, including sMPLC with the same histological type and sMPLC with different histological types. The demographic, clinical, pathological characteristics, and outcomes data of the included patients were collected and compared between the two groups. The ethics committee of our hospital approved this study and written informed consent was obtained from all participants.

2.2. Surgery Strategy. All enrolled patients underwent focal surgery resection plus systematic lymph node dissection and sampling. The surgery strategy was selected according to the size and location of lesions, the result of frozen section, the age, the pulmonary function, and basic physical condition of the patients [14]. Video-assisted thoracoscopic surgery was the main method. If the lesions were located on the ipsilateral side, they will be resected at the same time. Otherwise, staging operation was recommended. The surgery methods consisted of single lobectomy, multiple lobectomy, single sublobar resection, multiple sublobar resection, lobectomy plus sublobar resection, and total pneumonectomy. If the lesion was located in the same segment or same lobe, single sublobar resection and single lobectomy were used, respectively; when the lesions are located in different lung lobes, sublobar resections, lobectomies, and even total pneumonectomy were considered. In patients with multiple nodules with a dominant solid lesion, lobectomy of the dominant nodule along with sublobar resection of other nodules was the common procedure.

2.3. Clinical Assessment. All enrolled patients received a comprehensive assessment before surgery, including symptom inquiry, physical examination, laboratory test, chest radiograph, cardiopulmonary function test, chest and abdominal CT scan, brain MRI, and bone scan. Each tumor was staged separately according to the revised TNM system [15] and the most advanced disease stage of all tumors was used as final disease stage of the patient. The clinicopathologic data including age, gender, symptom, smoking history, respiratory function, laboratory test, tumor histology, tumor location, and size were investigated.

2.4. Molecular Analysis. Somatic mutations in epidermal growth factor receptor (EGFR) were tested with resected specimen using either amplification refractory mutation system (ARMS) including common EGFR mutations (covering 29 known mutations in exons 18-21) or the nextgeneration sequencing. Somatic mutations in Kirsten-rat sarcoma 2 viral oncogene homolog (KRAS) were tested with the next-generation sequencing. Immunohistochemistry was performed for detection of programmed cell death ligand 1 (PD-L1) and PD-L1 positive was defined as membranous staining present in $>1 \%$ of the cells. The anaplastic lymphoma kinase (ALK) and ROS proto-oncogene 1 (ROS1) immunohistochemistry assay were conducted and a binary scoring system was used to evaluate the staining results. The presence of strong granular cytoplasmic staining in tumor cells (any percentage of positive tumor cells) was considered as positive, and the absence of strong granular cytoplasmic staining in tumor cells was considered as negative.

2.5. Follow-Up. Patients were routinely followed up after surgery by telephone interview or clinic visit until 30 November 2019. Chest and abdominal CT scan was performed every 3 months from 1 to 2 years after operation, and every 6 months from 3 to 5 years after operation, and every 12 months after 5 years. The brain MRI and bone scan could be added according to the changes of the patient's condition. The overall survival (OS) was estimated from the date of surgery until death of any cause or the date of last follow-up. Recurrence-free survival (RFS) was defined as the time from the date of surgery to the first event, including recurrence and metastasis, or last follow-up. 


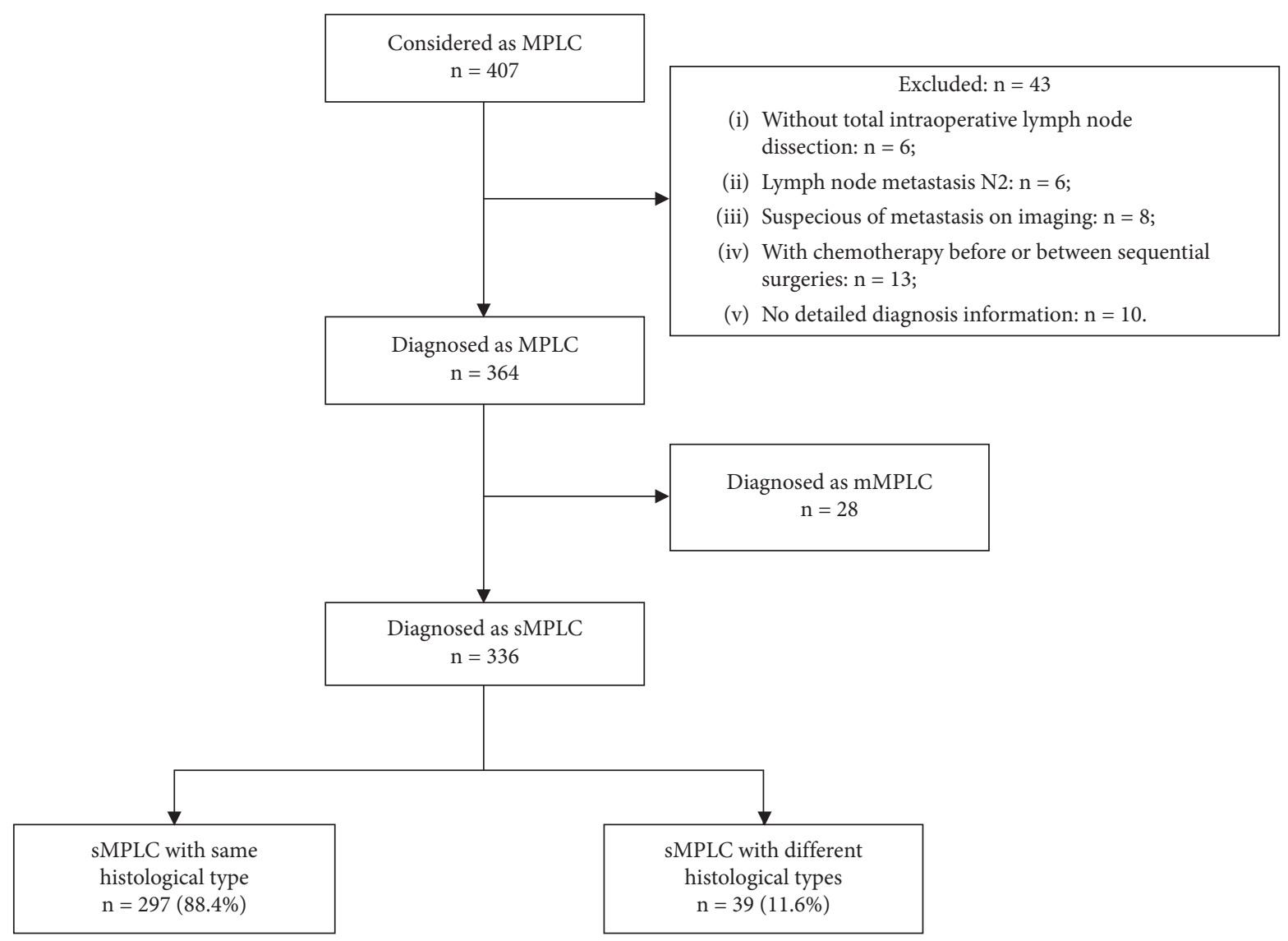

FIGURE 1: Flow chart. MPLC = multiple primary lung cancer; mMPLC $=$ metachronous multiple primary lung cancer; $s M P L C=s i-$ multaneous multiple primary lung cancer.

2.6. Statistical Analysis. Continuous variables were presented as median (interquartile range (IQR)) and compared using the Mann-Whitney $U$ test. Categorical variables were summarized by number (proportion) and compared with chi-square test or Fisher exact test. The OS curves and RFS were plotted using Kaplan-Meier method and compared with the log-rank test. A multivariable Cox proportional hazard model was used to evaluate potential factors associated with OS and RFS. Variables which demonstrate significant association with the outcome in univariable analysis were candidates for further multivariable analysis. Variable selection in final parsimonious multivariable model was based on a forward-stepwise selection procedure. In all the analyses, a 2 -tailed $p$ value $<0.05$ was considered statistically significant. All data were analyzed by using Statistic Package for Social Science (SPSS Inc., Chicago, USA) version 23.

\section{Results}

3.1. Enrolled Patients. From June 2010 to June 2019, a total of 407 patients were considered as the diagnosis of MPLC. Forty-three patients were excluded, thus 364 patients were definitely diagnosed as MPLC. Among patients with MPLC, 28 were mMPLC. Finally, 336 sMPLC patients were enrolled. Among the enrolled patients, multiple lesions with the same histological type were recorded in 297 patients
(88.4\%), whereas multiple lesions with different histological types were detected in the other 39 patients $(11.6 \%)$ (Figure 1).

3.2. Baseline Characteristics. The baseline clinical and histological characteristics are shown in Table 1 and Table 2, respectively. The median age of overall patients was 63 (56-70) years old and female was dominant (61.6\%). Most patients $(69.0 \%)$ were diagnosed with no symptoms and a smoking history was detected in $88(26.2 \%)$ patients. A total of $246(73.2 \%)$ patients had 2 lesions and only $13(3.9 \%)$ patients had more than 4 lesions. For histological characteristics, adenocarcinoma was the most common histological type, and most were ranging from stage I A to I B according to TNM staging.

Compared with patients with the same histological type, patients with different histological types were older during diagnosis (65 [62-69] vs. 59 [52-65]; $p<0.001)$ and less commonly females $(12.8 \%$ vs. $68.0 \% ; p<0.001)$. In addition, more patients with different histological types suffered from respiratory symptom and/or pain at diagnosis $(56.4 \%$ vs. $27.6 \%$; $p<0.001)$. Also, smoking history was more common in patients with different histological types (79.5\% vs. $16.8 \%$; $p<0.001)$. Importantly, patients with different histological types showed worse respiratory function with lower forced expiratory volume in 1 second (FEV1) and diffusing capacity 
TABLE 1: Baseline clinical characteristics.

\begin{tabular}{|c|c|c|c|c|}
\hline & Overall patients, $N=336$ & Same histological type, $N=297$ & Different histological types, $N=39$ & $p$ value \\
\hline \multicolumn{5}{|l|}{ Demographics } \\
\hline Age, yrs & $63(56-70)$ & $59(52-65)$ & $65(62-69)$ & $<0.001$ \\
\hline Females & $207(61.6)$ & $202(68.0)$ & $5(12.8)$ & $<0.001$ \\
\hline BMI, $\mathrm{kg} / \mathrm{m}^{2}$ & $22.9(20.9-24.7)$ & $22.8(20.8-24.7)$ & $23.8(21.1-25.0)$ & 0.315 \\
\hline \multicolumn{5}{|l|}{ Symptoms } \\
\hline No symptom & $232(69.0)$ & $215(72.4)$ & $17(43.6)$ & $<0.001$ \\
\hline Respiratory symptom & $72(21.4)$ & $58(19.5)$ & $14(35.8)$ & 0.019 \\
\hline Pain & $21(6.3)$ & $17(5.7)$ & $4(10.3)$ & 0.285 \\
\hline Both & $11(3.3)$ & $7(2.4)$ & $4(10.3)$ & 0.028 \\
\hline Smoking history & $88(26.2)$ & $50(16.8)$ & $31(79.5)$ & $<0.001$ \\
\hline \multicolumn{5}{|l|}{ Lung function (\%) } \\
\hline FEV1 & $102(90-113)$ & $104(94-114)$ & $89(72-96)$ & $<0.001$ \\
\hline DLCO & $100(86-112)$ & $102(88-112)$ & $85(80-97)$ & $<0.001$ \\
\hline \multicolumn{5}{|l|}{ Laboratory test } \\
\hline $\mathrm{LDH}$ & $158(139-176)$ & $158(139-176)$ & $155(143-180)$ & 0.617 \\
\hline dNLR & $1.47(1.17-1.84)$ & $1.44(1.14-1.82)$ & $1.73(1.38-1.99)$ & 0.013 \\
\hline Neutrophile granulocyte, $10^{9}$ & $3.16(2.57-4.05)$ & $3.11(2.55-3.90)$ & $4.02(2.92-4.92)$ & 0.002 \\
\hline \multicolumn{5}{|l|}{ Features of lesions } \\
\hline Number & & & & 0.085 \\
\hline 2 & $246(73.2)$ & $211(71.0)$ & $35(89.7)$ & \\
\hline 3 & $57(17.0)$ & $53(17.8)$ & $4(10.3)$ & \\
\hline 4 & $20(6.0)$ & $20(6.8)$ & $0(0)$ & \\
\hline$>4$ & $13(3.9)$ & $13(4.4)$ & $0(0)$ & \\
\hline \multicolumn{5}{|l|}{ Largest nodule size } \\
\hline$>2 \mathrm{~cm}$ & $156(46.4)$ & $120(40.4)$ & $36(92.3)$ & $<0.001$ \\
\hline$>1 \mathrm{~cm}$ & $294(87.5)$ & $255(85.9)$ & $39(100)$ & 0.008 \\
\hline Location & & & & 0.541 \\
\hline Ipsilateral & $209(62.2)$ & $183(61.6)$ & $26(66.7)$ & \\
\hline Bilateral & $127(37.8)$ & $114(38.4)$ & $13(33.3)$ & \\
\hline
\end{tabular}

Values are $n(\%)$ or median (interquartile range). BMI = body mass index; DLCO = diffusing capacity for carbon monoxide; dNLR=derive neutrophil-tolymphocyte radio; $\mathrm{FEV} 1$ = forced expiratory volume in 1 second; $\mathrm{LDH}=$ lactate dehydrogenase.

for carbon monoxide (DLCO). The inflammation biomarkers were analyzed, including serum lactate dehydrogenase (LDH), neutrophile granulocyte, and derive neutrophil-to-lymphocyte radio (dNLR). Patients with different histological types showed higher dNLR and neutrophile granulocytes. Interestingly, patients with different histological types had more pleural invasion $(56.4 \%$ vs. $29.0 \% ; p<0.001)$ and showed a more advanced stage according to TNM staging $(p<0.001)$.

3.3. Surgical Strategy. The surgical strategy is summarized in Supplemental Table 1. Only 74 (22.0\%) patients received sequential surgeries and the median surgery interval was 7 [4-17] months. Most patients (73.5\%) received thoracoscopic surgery and 18 (5.4\%) patients received both thoracoscopic surgery and open heart surgery. Lobectomy plus sublobar resection $(52.4 \%)$ was the most common surgery method, followed by multiple sublobar resection (34.8\%). There was no difference in surgical strategy between patients with the same histological type and different histological types.

3.4. Molecular Status. Mutations in EGFR were tested in 291 lesions of 196 patients (58.3\%) and the positive rate was 62.2\%. Among the 181 EGFR positive lesions, L858 R missense mutation was the most common site (112, 61.9\%), followed by exon 19 deletions (44, 24.3\%). Concomitance of two or three EGFR mutations were present in 13 lesions. The detailed information of EGFR mutation is shown in Supplemental Table 2. In addition, the positive rates of ALK, ROS1, KRAS, and PD-L1 were $2.0 \%, 11.3 \%, 5.3 \%$, and $11.6 \%$, respectively (Table 3 ). Interestingly, patients with the same histology had a higher positive rate of EGFR $(p=0.001)$ and ROS1 $(p=0.01)$ and lower positive rate of PD-L1 $(p=0.004)$.

3.5. Survival and Risk Factors of Overall Survival and Recurrence-Free Survival. Patients were followed up for a median period of 20 (11-34) months and 5 patients were lost to follow-up. During follow-up, 16 patients died, including 4 perioperative deaths and 12 deaths due to disease progression. The 1- and 3-year OS of overall patients were $97.7 \%$ and $92.2 \%$ (Figure 2(a)). The 1- and 3-year OS were $99.2 \%$ and $96.8 \%$ for patients with the same histological type, and $88.5 \%$ and $64.2 \%$ for patients with different histological types $(p<0.001)$ (Figure 3(a)). A total of 32 patients had recurrence. The 1- and 3-year RFS of overall patients were $96.8 \%$ and $85.7 \%$ (Figure 2(b)). The 1- and 3-year RFS were $97.8 \%$ and $89.0 \%$ for patients with the same histological type, and $88.1 \%$ and $62.1 \%$ for patients with different histological types $(p=0.002)$ (Figure 3(b)). 
TABle 2: Histological characteristics.

\begin{tabular}{|c|c|c|c|c|}
\hline & Overall patients, $N=336$ & Same histological type, $N=297$ & Different histological types, $N=39$ & $p$ value \\
\hline \multicolumn{5}{|l|}{ Pathologic characteristics } \\
\hline All adenocarcinoma & $291(86.6)$ & $291(98.0)$ & $0(0)$ & - \\
\hline & & & Adenocarcinoma + squamous & $26(7.7)$ \\
\hline $0(0)$ & $26(66.7)$ & - & & \\
\hline All squamous & $6(1.8)$ & $6(2.0)$ & $0(0)$ & - \\
\hline Adenocarcinoma + others & $11(3.3)$ & $0(0)$ & $11(28.2)$ & - \\
\hline Squamous + others & $2(0.6)$ & $0(0)$ & $2(5.1)$ & - \\
\hline TNM stage & & & & $<0.001$ \\
\hline I A1 & $63(18.8)$ & $63(21.2)$ & $0(0)$ & \\
\hline I A2 & $96(28.6)$ & $94(31.6)$ & $2(5.1)$ & \\
\hline I A3 & $46(13.7)$ & $42(14.1)$ & $4(10.2)$ & \\
\hline I B & $83(24.7)$ & $78(26.3)$ & $5(12.8)$ & \\
\hline II A & $12(3.6)$ & $9(3.0)$ & $3(7.7)$ & \\
\hline II B & $17(5.1)$ & $9(3.0)$ & $8(20.5)$ & \\
\hline III A & $13(3.9)$ & $2(0.7)$ & $11(28.2)$ & \\
\hline III B & $4(1.2)$ & $0(0)$ & $4(10.2)$ & \\
\hline IV A & $2(0.6)$ & $0(0)$ & $2(5.1)$ & \\
\hline Pleural invasion & $108(32.1)$ & $86(29.0)$ & $22(56.4)$ & 0.001 \\
\hline \multicolumn{5}{|l|}{ Treatment } \\
\hline Surgery timing & & & & 0.287 \\
\hline Concurrent & $262(78.0)$ & $229(77.1)$ & $33(84.6)$ & \\
\hline Sequential & $74(22.0)$ & $68(22.9)$ & $6(15.4)$ & \\
\hline Surgery interval, mon & 7 [4-17] & $7[4-16]$ & 9 [3-17] & 0.564 \\
\hline Resect all nodules & & & & 0.263 \\
\hline Yes & $122(36.3)$ & $111(37.4)$ & $11(28.2)$ & \\
\hline No & $214(63.7)$ & $186(62.6)$ & $28(71.8)$ & \\
\hline \multicolumn{5}{|l|}{ Other treatments } \\
\hline Chemotherapy & $135(40.2)$ & $109(36.7)$ & $26(66.6)$ & $<0.001$ \\
\hline Target therapy & $11(3.3)$ & $11(3.7)$ & $0(0)$ & 0.624 \\
\hline
\end{tabular}

Values are $n$ (\%) or median (interquartile range).

Table 4 summarizes the univariable and multivariable analysis of clinicopathologic factors related to OS. In univariable analyses, ten variables were associated with OS. Four significant predictors were retained, following forward-stepwise variable selection, in the final multivariate Cox regression model. The factors associated with a worse OS were the presence of different histological types (adjusted hazard ratio (HR): 10.00 ; 95\% CI: 2.92-34.48; $p<0.001$ ), the older age at diagnosis (adjusted HR: 1.14; 95\% CI: $1.04-1.26 ; p=0.008$ ), pleural invasion (adjusted HR: 7.09; 95\% CI: 1.42-35.71; $p=0.017)$, and elevated neutrophile granulocyte (adjusted HR: 2.06; 95\% CI: $1.30-3.27 ; p=0.002$ ).

Table 5 summarizes the univariable and multivariable analysis of clinicopathologic factors associated with RFS. In univariable analyses, nine variables were associated with RFS. Four significant predictors were retained, following forward-stepwise variable selection, in the final multivariate Cox regression model. The factors associated with a worse RFS were the presence of different histological types (adjusted HR: 2.59; 95\% CI: 1.14-5.88; $p=0.023$ ), the older age (adjusted HR: 1.06; 95\% CI: $1.01-1.11 ; p=0.023$ ), pleural invasion (adjusted HR: 2.36; 95\% CI: 1.07-5.18; $p=0.032$ ), and elevated neutrophile granulocyte (adjusted HR: 1.30; 95\% CI: 1.01-1.69; $p=0.032$ ).

\section{Discussion}

In this study, we conducted an analysis of clinicopathologic characteristics and outcomes in 336 sMPLC patients. We demonstrated that in sMPLC, patients with different histological types only accounted for $11.6 \%$. Importantly, although less common, patients with different histological types showed worse clinical characteristics and outcomes.

In patients with sMPLC, the histological types of different lesions are critical and some studies previously reported that patients with different histological types accounted for a proportion of $3.8 \%-61.5 \%$ [10-13]. However, the previous studies all had limited sample sizes. Our study with a relatively large cohort showed that patients with different histological types only accounted for $11.6 \%$ in sMPLC. It was speculated that the difference might be caused by the following reasons: different enrollment methods, regions, health awareness of residents, the popularity of physical examination, and surgical opportunities caused by the latest stage of sMPLC.

Remarkably, patients with different histological types showed an older age at diagnosis, more commonly males, more commonly with a smoking history and worse respiratory function than patients with the same histological type. These results hinted that the underlying mechanisms might be different between them. It was estimated that $75 \%$ of all 
TABLe 3: Molecular status.

\begin{tabular}{|c|c|c|c|c|}
\hline & All patients, $N=336$ & Same histological type, $N=297$ & Different histological types, $N=39$ & $p$ value \\
\hline \multicolumn{5}{|l|}{ EGFR } \\
\hline Tested patients & $196(58.3)$ & $180(60.6)$ & $16(41.0)$ & - \\
\hline Tested lesions & 291 & 268 & 23 & - \\
\hline EGFR+ & $181(62.2)$ & $174(64.9)$ & $7(30.4)$ & 0.001 \\
\hline EGFR- & $110(37.8)$ & $94(35.1)$ & $16(69.6)$ & - \\
\hline \multicolumn{5}{|l|}{ ALK } \\
\hline Tested patient & $290(86.3)$ & $257(86.5)$ & $33(84.6)$ & - \\
\hline Tested lesions & 450 & 403 & 47 & - \\
\hline ALK+ & $9(2.0)$ & $8(2.0)$ & $1(2.1)$ & 0.947 \\
\hline ALK- & $441(98.0)$ & $395(98.0)$ & $46(97.9)$ & - \\
\hline \multicolumn{5}{|l|}{ ROS1 } \\
\hline Tested patient & $280(83.3)$ & $248(83.5)$ & $32(82.1)$ & - \\
\hline Tested lesions & 433 & 387 & 46 & - \\
\hline ROS1+ & $49(11.3)$ & $49(12.7)$ & $0(0)$ & 0.01 \\
\hline ROS1- & $231(88.7)$ & $338(87.3)$ & $46(100)$ & - \\
\hline \multicolumn{5}{|l|}{ KRAS } \\
\hline Tested patient & $35(10.4)$ & $33(11.1)$ & $2(5.1)$ & - \\
\hline Tested lesions & 57 & 54 & 3 & - \\
\hline KRAS+ & $3(5.3)$ & $3(5.6)$ & $0(0)$ & 0.675 \\
\hline KRAS- & $54(94.7)$ & $51(94.4)$ & $3(100)$ & - \\
\hline \multicolumn{5}{|l|}{ PD-L1 } \\
\hline Tested patient & $190(56.5)$ & $172(57.9)$ & $18(46.2)$ & - \\
\hline Tested lesions & 285 & 259 & 26 & - \\
\hline PD-L1+ & $33(11.6)$ & $25(9.7)$ & $8(30.8)$ & 0.004 \\
\hline PD-L1- & $252(88.4)$ & $234(90.3)$ & $18(69.2)$ & - \\
\hline
\end{tabular}

ALK = anaplastic lymphoma kinase; EGFR = epidermal growth factor receptor; KRAS=Kirsten-rat sarcoma 2 viral oncogene homolog; PD$\mathrm{L} 1=$ programmed cell death ligand 1; ROS1 = ROS proto-oncogene 1.

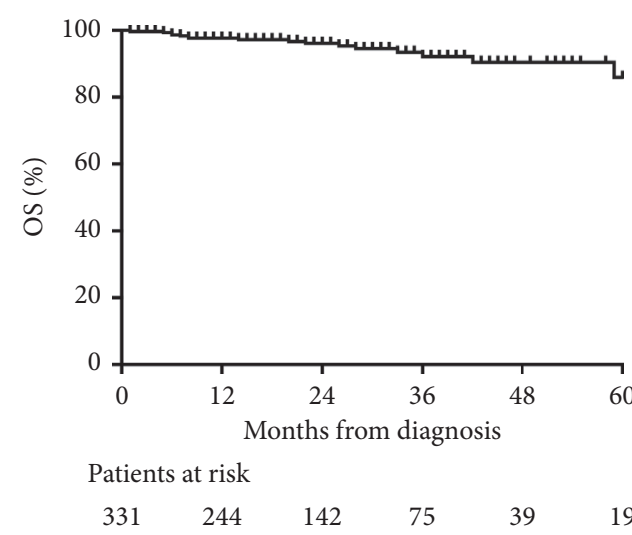

(a)

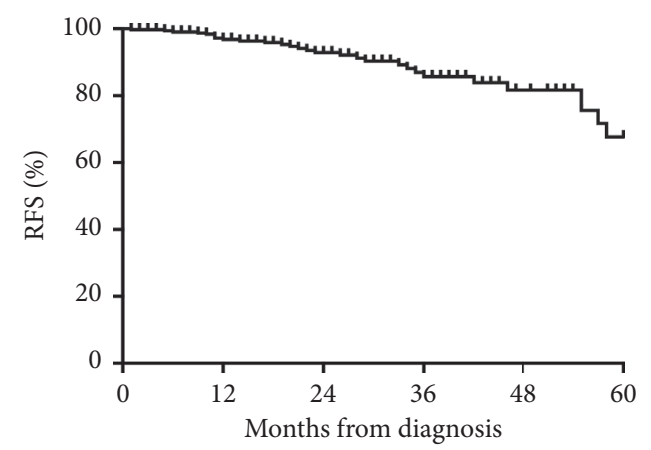

Patients at risk

$\begin{array}{llllll}331 & 238 & 133 & 70 & 36 & 14\end{array}$

(b)

FIGURE 2: OS and RFS of overall patients. OS = overall survival; RFS = recurrence-free survival.

lung cancers worldwide are smokers, consisting of $85 \%$ in men and $47 \%$ in women [16]. However, the rates of smokers in sMPLC seem to be lower and vary from $32.3 \%$ to $59.0 \%$ $[12,17,18]$. Furthermore, some studies reported that the rate of smokers in patients with multiple ground-glass nodules was much lower than that in patients with multiple solid nodules $[18,19]$. All these findings suggested that smoking might have a lower impact on the development of sMPLC, especially for those with multiple ground-glass nodules. In our study, the rate of smokers was $26.2 \%$ in overall sMPLC, $16.8 \%$ in patients with the same histological type, and $79.5 \%$ in patients with different histological types. The relative low rate of smokers in our study was due to the major patients with the same histological type, which includes the majority of multiple ground-glass nodules. The different rates of smoking history between these two groups also suggest that smoking might not be the pathogenesis in patients with sMPLC with the same histology, but contribute to the development of sMPLC with different histological types.

The inflammatory process has been considered as an important mechanism of immune resistance, tumor growth and proliferation, and activation of cancer signaling pathway in cancer patients. Peripheral inflammatory state is related to poor prognosis of cancer patients [20]. A large number of 


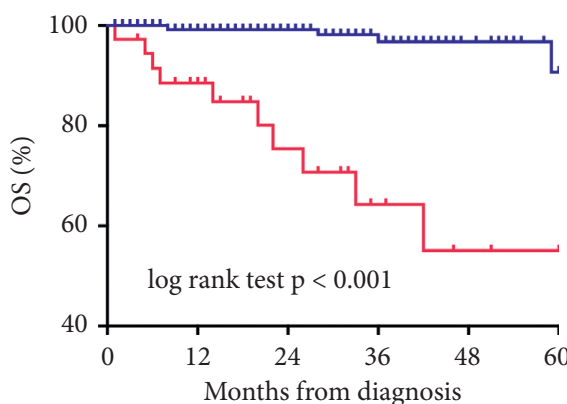

Patients at risk

$\begin{array}{lllllll}\text { Same histological type } & 294 & 218 & 125 & 65 & 33 & 15\end{array}$

Different histological types $37 \quad 26 \quad 17 \quad 10 \quad 6 \quad 4$

\section{- Same histological type}

_ Different histological types

(a)

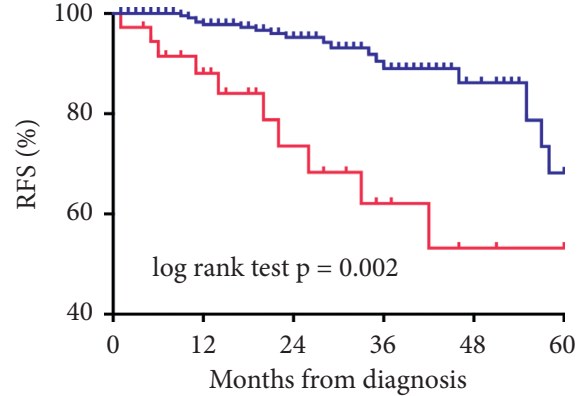

Patients at risk

\begin{tabular}{lcccccc} 
Same histological type & 294 & 214 & 118 & 60 & 30 & 10 \\
Different histological types & 37 & 24 & 15 & 10 & 6 & 4 \\
& \multicolumn{5}{c}{ Same histological type } \\
& \multicolumn{4}{c}{ Different histological types }
\end{tabular}

(b)

FIGURE 3: OS and RFS of patients with the same histological type and different histological types. OS = overall survival; RFS = recurrence-free survival.

TABLE 4: Univariable and multivariable analyses of clinicopathologic factors associated with OS.

\begin{tabular}{|c|c|c|c|c|}
\hline \multirow{2}{*}{ Characteristics } & \multicolumn{2}{|c|}{ Univariable analysis* } & \multicolumn{2}{|c|}{ Multivariable analysis $^{\dagger}$} \\
\hline & HR (95\% CI) & $p$ value & HR $(95 \% \mathrm{CI})$ & $p$ value \\
\hline Age, yrs & $1.10(1.03-1.18)$ & 0.005 & $1.14(1.04-1.26)$ & 0.008 \\
\hline Females & $10.07(2.28-44.49)$ & 0.002 & & \\
\hline Histology, different vs. same & $12.29(4.44-34.03)$ & $<0.001$ & $10.00(2.92-34.48)$ & $<0.001$ \\
\hline Smoking history & $19.12(4.33-84.34)$ & $<0.001$ & & \\
\hline FEV1 & $0.98(0.95-0.99)$ & 0.033 & & \\
\hline DLCO & $0.99(0.96-1.01)$ & 0.333 & & \\
\hline dNLR & $1.82(1.10-3.02)$ & 0.021 & & \\
\hline Neutrophile granulocyte, $10^{9}$ & $1.97(1.42-2.73)$ & $<0.001$ & $2.06(1.30-3.27)$ & 0.002 \\
\hline Number of lesions, $>2$ vs. 2 & $1.01(0.32-3.18)$ & 0.987 & & \\
\hline Largest nodule size, $>2$ vs. $<2 \mathrm{~cm}$ & $5.05(1.43-17.81)$ & 0.012 & & \\
\hline TNM stage, IB-IV vs. IA & $7.87(1.77-34.48)$ & 0.007 & & \\
\hline Pleural invasion & $9.43(2.10-41.67)$ & 0.003 & $7.09(1.42-35.71)$ & 0.017 \\
\hline
\end{tabular}

$\mathrm{BMI}=$ body mass index; $\mathrm{DLCO}$ = diffusing capacity for carbon monoxide; $\mathrm{dNLR}=$ derive neutrophil-to-lymphocyte radio; FEV1 = forced expiratory volume in 1 second; $\mathrm{LDH}=$ lactate dehydrogenase; $\mathrm{OS}$ = overall survival.

TABLE 5: Univariable and multivariable analyses of clinicopathologic factors associated with RFS.

\begin{tabular}{|c|c|c|c|c|}
\hline \multirow{2}{*}{ Characteristics } & \multicolumn{2}{|c|}{ Univariable analysis* } & \multicolumn{2}{|c|}{ Multivariable analysis $^{\dagger}$} \\
\hline & $\operatorname{HR}(95 \% \mathrm{CI})$ & $p$ value & HR $(95 \%$ CI $)$ & $p$ value \\
\hline Age, yrs & $1.06(1.02-1.11)$ & 0.010 & $1.06(1.01-1.11)$ & 0.023 \\
\hline Females & $2.10(1.02-4.34)$ & 0.045 & & \\
\hline Histology, different vs. same & $2.96(1.39-6.33)$ & 0.005 & $2.59(1.14-5.88)$ & 0.023 \\
\hline Smoking history & $2.29(1.14-4.63)$ & 0.020 & & \\
\hline FEV1 & $1.00(0.99-1.02)$ & 0.855 & & \\
\hline DLCO & $1.01(0.99-1.03)$ & 0.314 & & \\
\hline dNLR & $1.59(1.05-2.40)$ & 0.028 & & \\
\hline Neutrophile granulocyte, $10^{9}$ & $1.47(1.14-1.89)$ & 0.003 & $1.30(1.01-1.69)$ & 0.046 \\
\hline Number of lesions, $>2$ vs. 2 & $1.69(0.82-3.47)$ & 0.156 & & \\
\hline Largest nodule size, $>2$ vs. $<2 \mathrm{~cm}$ & $2.27(1.09-4.72)$ & 0.028 & & \\
\hline TNM stage, IB-IV vs. IA & $2.60(1.19-5.68)$ & 0.016 & & \\
\hline Pleural invasion & $2.81(1.31-6.02)$ & 0.008 & $2.36(1.07-5.18)$ & 0.032 \\
\hline
\end{tabular}

$\mathrm{BMI}=$ body mass index; $\mathrm{DLCO}$ = diffusing capacity for carbon monoxide; $\mathrm{dNLR}=$ derive neutrophil-to-lymphocyte radio; FEV1 = forced expiratory volume in 1 second; $\mathrm{LDH}=$ lactate dehydrogenase; RFS = recurrence-free survival. 
routine blood parameters have been studied as potential biomarkers of inflammation in cancer patients, such as the increase of circulating leukocyte concentration, neutrophil absolute number, dNLR, and LDH level [21]. In this study, we demonstrated that dNLR and neutrophil absolute number were higher in patients with different histological types, and elevated neutrophil absolute value was significant predictor for worse OS and RFS, which indicated that inflammatory process might have a more important role in patients with different histological types.

Previous studies have revealed that large tumor size and lymph node involvement were independent factors for worse survival [22-24]. However, in our study, we initially reported that presence of different histological types was independent factors for worse OS and RFS. Large tumor size and advanced TNM stage were all significant in univariable analysis, but not significant in multivariable analysis. Instead, pleural invasion, another important parameter indicating disease progression, was independent factor for worse OS and RFS. For the overall patients in our study, the prognosis was relatively good after surgical resection compared to patients with metastatic and recurrent cancer $[25,26]$, which emphasized the importance for accurate diagnosis in clinical practice. However, patients with different histological types showed an obviously worse OS and RFS than patients with the same histological type and the presence of different histological types was significant predictor for worse OS and RFS. This might be explained by the following reasons. First, as discussed above, the underlying mechanisms between the two groups might be different, and inflammatory process and smoking had a more significant role in patients with different histological types. Second, patients with different histological types were diagnosed at an older age. Third, patients with different histological types showed worse clinical status at baseline with worse respiratory function and advanced stages. Due to the distinct OS and RFS between them, it is essential to distinguish them in clinical practice.

This study had some limitations. First, both Martini-Melamed and American College of Chest Physicians criteria are based on clinicopathological and radiological features of lung nodules. Despite their convenience to apply, they have notable limitations in the recognition of multiple intrapulmonary metastases. The forefront molecular approaches suggest their superiorities in distinguishing between MPLC and intro-pulmonary metastasis and there was a lack of in-depth molecular analysis of multiple lung lesions in this work. Second, this study was a single-center study. Third, due to the differences in clinical characteristics and genomics between Chinese and Western populations, our results need to be further studied and validated in Western patients. Last, the follow-up time span was not long enough and the numbers of events were relatively limited. Although the effect value was strong and statistically significant, considering that the wide confidence interval reflected the instability of the results, the interpretation of the results needed to be cautious and confirmed by further researches.

\section{Conclusions}

We demonstrated that in sMPLC, patients with different histological types only accounted for $11.6 \%$. Importantly, although less common, patients with different histological types showed more severe clinical characteristics and lower recurrence-free survival and overall survival than patients with the same histological type.

\section{Data Availability}

The data used to support the findings of this study are available from the corresponding author upon request.

\section{Disclosure}

The abstract of this paper was presented at the 2020 ASCO Conference as online publication.

\section{Conflicts of Interest}

All authors have no conflicts of interest to disclose.

\section{Authors' Contributions}

Ying Liu and Yu-Ping Zhou contributed equally to this article.

\section{Acknowledgments}

The authors gratefully thank the patients for their involvements in this study. This work was supported by the 1.3.5 Project for Disciplines of Excellence-Clinical Research Incubation Project, West China Hospital, Sichuan University (Grant no. 2019HXFH062).

\section{Supplementary Materials}

Supplementary Table 1. Surgical strategy. Supplemental Table 2. Detailed information of EGFR mutation. (Supplementary Materials)

\section{References}

[1] N. Martini and M. R. Melamed, "Multiple primary lung cancers," The Journal of Thoracic and Cardiovascular Surgery, vol. 70, no. 4, pp. 606-612, 1975.

[2] K. R. Shen, B. F. Meyers, J. M. Larner, D. R. Jones, and American College of Chest Physicians, "Special treatment issues in lung cancer," Chest, vol. 132, no. 3, pp. 290S-305S, 2007.

[3] Y. Liu, J. Zhang, L. Li et al., "Genomic heterogeneity of multiple synchronous lung cancer," Nature Communications, vol. 7, no. 1, p. 13200, 2016.

[4] G. Pei, M. Li, X. Min et al., "Molecular identification and genetic characterization of early-stage multiple primary lung cancer by large-panel next-generation sequencing analysis," Frontiers in Oncology, vol. 11, p. 653988, 2021.

[5] H. Wang, J. Hou, G. Zhang et al., "Clinical characteristics and prognostic analysis of multiple primary malignant neoplasms in patients with lung cancer," Cancer Gene Therapy, vol. 26, no. 11-12, pp. 419-426, 2019. 
[6] M.-L. Audrey, B. Marc, and A. Marco, "Proposal for a combined histo-molecular algorithm to distinguish multiple primary adenocarcinomas from intrapulmonary metastasis in patients with multiple lung tumors," Journal of Thoracic Oncology, vol. 14, pp. 844-856, 2019.

[7] M. Vazquez, D. Carter, E. Brambilla et al., "Solitary and multiple resected adenocarcinomas after CT screening for lung cancer: histopathologic features and their prognostic implications," Lung Cancer, vol. 64, no. 2, pp. 148-154, 2009.

[8] T. Tanvetyanon and T. A. Boyle, "Clinical implications of genetic heterogeneity in multifocal pulmonary adenocarcinomas," Journal of Thoracic Disease, vol. 8, no. 12, pp. E1734-E1738, 2016.

[9] H. Asamura, "Multiple primary cancers or multiple metastases, that is the question," Journal of Thoracic Oncology, vol. 5, no. 7, pp. 930-931, 2010.

[10] Y. Yang, W. Yin, W. He et al., "Phenotype-genotype correlation in multiple primary lung cancer patients in China," Scientific Reports, vol. 6, no. 1, p. 36177, 2016.

[11] Y. Zhang, H. Hu, and R. Wang, "Synchronous non-small cell lung cancers: diagnostic yield can be improved by histologic and genetic methods," Annals of Surgical Oncology, vol. 21, pp. 4369-4374, 2014.

[12] K. Chen, W. Chen, J. Cai et al., "Favorable prognosis and high discrepancy of genetic features in surgical patients with multiple primary lung cancers," The Journal of Thoracic and Cardiovascular Surgery, vol. 155, pp. 371-e1, 2018.

[13] F. Xiao, D. Liu, Y. Guo et al., "Survival rate and prognostic factors of surgically resected clinically synchronous multiple primary non-small cell lung cancer and further differentiation from intrapulmonary metastasis," Journal of Thoracic Disease, vol. 9, no. 4, pp. 990-1001, 2017.

[14] B. D. Kozower, J. M. Larner, F. C. Detterbeck, and D. R. Jones, "Special treatment issues in non-small cell lung cancer: diagnosis and management of lung cancer, 3rd ed: American College of Chest Physicians evidence-based clinical practice guidelines," Chest, vol. 143, pp. e369S-e399S, 2013.

[15] F. C. Detterbeck, A. G. Nicholson, W. A. Franklin et al., "The IASLC Lung Cancer Staging Project: summary of proposals for revisions of the classification of lung cancers with multiple pulmonary sites of involvement in the forthcoming eighth edition of the TNM classification," Journal of Thoracic Oncology: Official Publication of the International Association for the Study of Lung Cancer, vol. 11, no. 5, pp. 639-650, 2016.

[16] D. M. Parkin, F. Bray, J. Ferlay, and P. Pisani, "Global cancer statistics, 2002," CA: A Cancer Journal for Clinicians, vol. 55, no. 74-108, 2005.

[17] Z. Zhang, S. Gao, Y. Mao et al., "Surgical outcomes of synchronous multiple primary non-small cell lung cancers," Scientific Reports, vol. 6, no. 1, p. 23252, 2016.

[18] R. W. Gao, M. F. Berry, C. A. Kunder et al., "Survival and risk factors for progression after resection of the dominant tumor in multifocal, lepidic-type pulmonary adenocarcinoma," The Journal of Thoracic and Cardiovascular Surgery, vol. 154, no. 6, pp. 2092-2099, 2017.

[19] Y. Zhang, G. Li, Y. Li et al., "Imaging features suggestive of multiple primary lung adenocarcinomas," Annals of Surgical Oncology, vol. 27, no. 6, pp. 2061-2070, 2020.

[20] F. Petrelli, M. Cabiddu, A. Coinu et al., "Prognostic role of lactate dehydrogenase in solid tumors: a systematic review and meta-analysis of 76 studies," Acta Oncologica, vol. 54, no. 7, pp. 961-970, 2015.

[21] A. Paramanathan, A. Saxena, and D. L. Morris, "A systematic review and meta-analysis on the impact of pre-operative neutrophil lymphocyte ratio on long term outcomes after curative intent resection of solid tumours," Surgical Oncology, vol. 23, no. 1, pp. 31-39, 2014.

[22] Z. Zhang, S. Gao, Y. Mao et al., "Surgical outcomes of synchronous multiple primary non-small cell lung cancers," Scientific Reports, vol. 6, no. 1, p. 23252, 2016.

[23] Y.-L. Chang, C.-T. Wu, and Y.-C. Lee, "Surgical treatment of synchronous multiple primary lung cancers: experience of 92 patients," The Journal of Thoracic and Cardiovascular Surgery, vol. 134, no. 3, pp. 630-637, 2007.

[24] J. Lv, D. Zhu, X. Wang, Q. Shen, Q. Rao, and X. Zhou, "The value of prognostic factors for survival in synchronous multifocal lung cancer: a retrospective analysis of 164 patients," The Annals of Thoracic Surgery, vol. 105, no. 3, pp. 930-936, 2018.

[25] Z. Zhang, S. Gao, Y. Mao et al., "Surgical outcomes of synchronous multiple primary non-small cell lung cancers," Scientific Reports, vol. 6, no. 1, p. 23252, 2016.

[26] L. Voltolini, C. Rapicetta, L. Luzzi et al., "Surgical treatment of synchronous multiple lung cancer located in a different lobe or lung: high survival in node-negative subgroup," European Journal of Cardio-Thoracic Surgery, vol. 37, no. 5, pp. 1198-1204, 2010. 